\title{
FACTORS ASSOCIATED WITH COVID-19 AND ASYMPTOMATIC CARRIAGE IN HEALTHCARE WORKERS OF A COVID-19 HOSPITAL
}

\author{
Eric Ochoa-Hein ${ }^{1}$, Sandra Rajme-López ${ }^{2}$, Patricia E. Leal-Morán ${ }^{1}$, Abril T. Vargas-Fernández ${ }^{1}$, \\ María F. González-Lara ${ }^{2}$, María F. Alberto-Hernández ${ }^{3}$, Daniel E. Bustos-Román ${ }^{3}$, \\ Lirio N. Valverde-Ramos ${ }^{3}$, Pilar Ramos-Cervantes ${ }^{2}$, Luz E. Cervantes-Villar ${ }^{2}$, \\ Violeta Ibarra-GonzÁlez ${ }^{2}$, Alfredo Ponce-de-León ${ }^{2}$, Arturo Galindo-Fraga ${ }^{1}$,

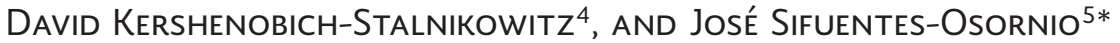 \\ Departments of ${ }^{1}$ Hospital Epidemiology and ${ }^{2}$ Infectious Diseases, Instituto Nacional de Ciencias Médicas \\ y Nutrición Salvador Zubirán (INCMyNSZ), Mexico City; ${ }^{3}$ General Direction of Epidemiology, Ministry of Health; \\ ${ }^{4}$ General Directorate, and ${ }^{5}$ Medicine Directorate, INCMyNSZ, Mexico City, Mexico
}

\begin{abstract}
Background: Risk factors for coronavirus disease (COVID-19) and severe acute respiratory syndrome coronavirus 2 (SARS-CoV-2) asymptomatic carriage ( $A C$ ) in healthcare workers (HCWs) have been scarcely characterized. Objective: The objective of the study was to study factors associated with COVID-19 and AC in HCWs of a COVID-19 academic medical center. Methods: This is a case-control study. Cases were either symptomatic or asymptomatic HCWs with a positive SARS-CoV-2 polymerase chain reaction (PCR) test result between March 16 and May 21 of 2020. Adjusted odds ratios (aOR) were calculated by means of multivariable logistic regression. In addition, each subject was followed for 14 days to inform outcomes. Results: One hundred thirty of 249 (52.2\%) symptomatic HCWs had COVID-19; 10 were hospitalized but none died. Of 987 asymptomatic HCWs, $37(3.7 \%)$ were AC; 6 of the remaining 950 asymptomatic HCWs with a negative PCR test result were found to be presymptomatic COVID-19 cases the following 14 days. Nurses were more frequently present in the COVID-19 group (51.5\% vs. $37.0 \%$ ), but multivariable analysis rendered non-significant results. After adjustment for age, comorbidities, and working place, factors found to be associated with AC were: working in wards as a nurse (aOR $=9.19,95 \%$ confidence interval $[\mathrm{Cl}]=1.05-80.22$, $p=0.045$ ), kitchen personnel $(\mathrm{aOR}=4.09,95 \% \mathrm{Cl}=1.55-10.83, \mathrm{p}=0.005$ ), and being a physician $(\mathrm{aOR}=0.12,95 \%$ $\mathrm{Cl}=0.03-0.54, p=0.006$ ). Conclusions: $\mathrm{HCW}$ category was the predominant factor associated with AC of SARS-CoV-2 in this study. (REV INVEST CLIN. 2021;73(2):65-71)
\end{abstract}

Key words: Coronavirus disease-19. Severe acute respiratory syndrome coronavirus 2. Healthcare worker. Carrier state. Risk factor.

*Corresponding author:

José Sifuentes-Osornio

E-mail: jose.sifuenteso@incmnsz.mx
Received for publication: 07-12-2020

Approved for publication: 19-01-2021

DOI: $10.24875 / R I C .20000592$

0034-8376 / (c) 2020 Revista de Investigación Clínica. Published by Permanyer. This is an open access article under the CC BY-NC-ND license (http://creativecommons.org/licenses/by-nc-nd/4.0/). 


\section{INTRODUCTION}

Coronavirus disease (COVID-19) is a newly identified viral disease that is present worldwide and is associated with an ever-increasing number of cases as well as deaths. Previous studies have reported a high risk of infection for healthcare workers (HCWs). Early in the pandemic, $1.1 \%$ of HCWs in Wuhan, China, were found to be infected as compared to $0.2 \%$ of the people living in the community ${ }^{1}$. In the United States and England, HCWs had a 12-fold higher risk of having a positive test compared to the general population ${ }^{2}$. In Mexico, HCWs accounted for $14.5 \%$ of total national confirmed cases as of November 23, 20203,4. Finally, prevalences of asymptomatic carriers (AC) have been estimated to be as high as $6.5 \%$ in $\mathrm{HCWs}^{1,5,6}$.

Identification of risk factors for being either a COVID-19 case or an AC is deemed important for control purposes (i.e., to halt transmission). Although it is generally assumed that HCWs in direct contact with COVID-19 patients (e.g., physicians and nurses) are the most at risk for COVID-19, some studies have found a higher risk in HCWs not working in clinical areas ${ }^{1,7,8}$. Interestingly, a higher incidence of infection in nurses working in non-clinical areas ${ }^{9}$ and a higher frequency of disease or antibody positivity in HCWs exposed to ill family members ${ }^{10}$, as noted by previous studies, point to associated factors present in the community. On the other hand, relatively little is known regarding factors associated with ACs; being a young woman ${ }^{9}$ and a HCW from COVID-19 healthcare centers ${ }^{6}$ were identified as risk factors in prior reports. The objective of this research was to study factors associated with COVID-19 and AC in HCWs of a COVID-19 academic medical center, as well as related outcomes.

\section{METHODS}

\section{Study design}

A case-control study was done. The need for informed consent was waived by the Institutional Review Board as this study was deemed non-experimental in nature.
Cases were defined as either symptomatic HCWs with a positive severe acute respiratory syndrome coronavirus 2 (SARS-CoV-2) polymerase chain reaction (PCR) test result (analysis \#1, factors associated with COVID-19), or asymptomatic HCWs with a positive $P C R$ test result (analysis \#2, factors associated with $A C$ ). Corresponding unmatched controls were symptomatic or asymptomatic HCWs with negative PCR test results, respectively. Only subjects with tests done between March 16 and May 21,2020 , were included in the analysis. All subjects were followed for 14 days after testing date to inform outcomes (hospitalization or death in HCWs with COVID-19, and progression to COVID-19 in ACs and HCWs with negative PCR test results). Asymptomatic and symptomatic subjects with initial negative test results were followed remotely through telephone calls without repeat tests, unless symptoms suggestive of COVID-19 appeared after 14 days of follow-up, in which case, they were treated as new suspect cases.

Symptomatic HCWs had at least one highly suggestive symptom (fever, headache, cough, or dyspnea) plus another symptom (malaise, myalgias, arthralgias, rhinorrhea, throat pain, conjunctivitis, vomiting, or diarrhea). Asymptomatic HCWs lacked any symptom and were identified from a database of a voluntary surveillance program that consisted of a 1-time questionnaire plus a free PCR test. The following data were recorded in the database: sex, age, comorbidities, worker category, working area, date of screening (asymptomatic $\mathrm{HCWs}$ ), and date of symptom onset (symptomatic HCWs).

\section{Sampling and testing for SARS-CoV-2}

Naso/oropharyngeal swab samples were obtained and sent to the laboratory in a universal transport medium for viruses. Nucleic acid extraction was done using the NucliSens easyMAG system (bioMérieux, Boxtel, Netherlands). Real-time reverse-transcription PCR was carried out in the Applied Biosystems 7500 thermocycler (Applied Biosystems, Foster City, (A, USA) using primers and conditions described elsewhere ${ }^{11}$; the cycle threshold value for positivity was 38 . 


\section{Statistical analysis}

Electronic databases were anonymized before conducting analyses and are available only to investigators of this study. Data were summarized using percentages for categorical variables, and medians and interquartile ranges for numerical data. Missing data were not replaced. For comparisons between groups, either the Chi-square test or Fisher's exact test were used for categorical variables, as appropriate; and Wilcoxon's rank sum test was used for numerical variables. Variables with a $p \leq 0.05$ were analyzed by logistic regression to identify factors associated with being a COVID-19 case or an AC; adjusted odds ratios (aORs) with their respective $95 \%$ confidence intervals ( $95 \%$ $\mathrm{Cl}$ ) were calculated after adjusting for variables previously mentioned (sex, age, comorbidities, worker category, and working area). Stata version 14.0 (StataCorp, College Station, TX, USA) was used for the aforementioned analyses.

\section{Epidemic curve}

The hospital's COVID-19 epidemic curve was compared to that reported for the Mexico City metropolitan area during the same time period ${ }^{12}$, using the date of symptom onset.

\section{Infection prevention and control measures}

After March 16, 2020, in line with hospital conversion, infection prevention and control policies were modified. First, use of personal protective equipment was mandatory for any HCW treating COVID-19 patients: universal use of a face mask, goggles, gowns, and gloves was instituted; surgical face masks were worn if treating patients hospitalized in wards, and N95/ KN95 respirators were used if treating patients in the Intensive Care Unit (ICU) or Emergency Room (ER) or if performing aerosolizing procedures (especially, endotracheal intubation or suction of airway secretions). Hand hygiene with alcohol hand rubs was especially enforced and was preferred over the use of $2 \%$ chlorhexidine soap. Each HCW was given a full kit with these items at the start of each working day, besides having access to extra equipment at the point of patient care. Universal use of face masks was not mandatory in non-clinical hospital areas during the study time period.

\section{RESULTS}

Of 249 symptomatic HCWs, 130 (52.2\%) were diagnosed with COVID-19. Of the symptomatic HCWs with negative PCR test results, no one was subsequently diagnosed with COVID-19 at the end of the 14-day follow-up. Of 987 asymptomatic HCWs, 37 (3.7\%) were positive to SARS-CoV-2, and none of the latter were diagnosed with COVID-19 after completing the 14-day follow-up. Of $950 \mathrm{HCWs}$ with initial negative test results, $6(0.6 \%)$ were later diagnosed with COVID-19 a median of 11 days (range, 5-19 days) after screening. Therefore, there were 136 COVID-19 cases and 37 ACs that were compared to 119 symptomatic and 944 asymptomatic controls, respectively.

Ten out of 136 (7.4\%) HCWs with COVID-19 were admitted to hospitalization (median length of stay = 9 days; interquartile range $=8-10$ days) and ultimately discharged alive without the need for admission to the ICU or assisted mechanical ventilation.

Of the 1236 subjects included in this study, $41.8 \%$ were male and the median age was 34 years (interquartile range $=28-45)$. Physicians $(35.4 \%)$ and nurses $(31.8 \%$ ) comprised the majority of the study population. Systemic hypertension (4.1\%) was the most prevalent comorbidity, followed by lung disease $(2.8 \%)$, which included asthma and chronic obstructive pulmonary disease. The majority of subjects $(30.1 \%)$ worked in the ICU or the ER, and $20.0 \%$ worked in non-clinical areas. The vast majority lived in Mexico City; $9.7 \%$ of subjects lived in other states.

Nurses were more prevalent in the group of COVID-19 cases according to bivariate analysis \#1 (Table 1). However, this finding lost statistical significance in a multivariable analysis after adjusting for age, comorbidities, and working place (aOR $=1.44$, $95 \% \mathrm{Cl}=0.74-2.79, \mathrm{p}=0.283$ ).

Bivariate analysis \#2 showed significant differences in the proportion of nurses working in wards, kitchen 
Table 1. Factors associated with being a COVID-19 case in bivariate analysis

\begin{tabular}{|c|c|c|c|c|}
\hline Variable & $\begin{array}{c}\text { Cases } \\
(n=136)^{*}\end{array}$ & $\begin{array}{l}\text { Controls } \\
(n=119)^{*}\end{array}$ & $\begin{array}{c}\text { OR } \\
(95 \% \mathrm{Cl})\end{array}$ & $\mathrm{p}$ \\
\hline Male sex & $52(38.2)$ & $47(39.5)$ & $0.95(0.56-1.62)$ & 0.837 \\
\hline Age, years (median, IQR) & $37(28.6-46.8)$ & $36(29.5-42)$ & $1.01(0.99-1.04)$ & 0.355 \\
\hline \multicolumn{5}{|l|}{ Working category } \\
\hline Physician & $29(21.3)$ & $35(29.4)$ & $0.65(0.35-1.19)$ & 0.137 \\
\hline Nurse & $70(51.5)$ & $44(37.0)$ & $1.81(1.06-3.08)$ & 0.020 \\
\hline Laboratory worker & $4(2.9)$ & $2(1.7)$ & $1.77(0.25-19.89)$ & 0.508 \\
\hline Kitchen personnel & $1(0.7)$ & $1(0.8)$ & $0.87(0.01-69.18)$ & 0.924 \\
\hline Office personnel & $9(6.6)$ & $16(13.5)$ & $0.46(0.17-1.15)$ & 0.067 \\
\hline Other category & $23(17.0)$ & $21(17.6)$ & $0.95(0.47-1.93)$ & 0.877 \\
\hline \multicolumn{5}{|l|}{ Comorbidities } \\
\hline Diabetes mellitus & $6 / 133(4.5)$ & $1(0.8)$ & $5.57(0.66-258.51)$ & 0.077 \\
\hline Lung disease & $1 / 133(0.8)$ & $5(4.2)$ & $0.17(0.0-1.59)$ & 0.073 \\
\hline Systemic hypertension & $8 / 133(6.0)$ & $10(8.4)$ & $0.70(0.23-2.04)$ & 0.462 \\
\hline Cardiovascular disease & $0 / 133(0)$ & $1(0.8)$ & 0 & 0.290 \\
\hline Obesity (BMI $\geq 30$ ) & $19 / 121(15.7)$ & $17 / 117(14.5)$ & $1.10(0.51-2.39)$ & 0.801 \\
\hline Current smoker & $4 / 133(3.0)$ & $5(4.2)$ & $0.71(0.14-3.38)$ & 0.610 \\
\hline \multicolumn{5}{|l|}{ Working place } \\
\hline ICU/ER & $15 / 130(11.5)$ & $5 / 111(4.5)$ & $2.77(0.91-10.03)$ & 0.049 \\
\hline Wards (Nurses) & $50 / 130(38.5)$ & $36 / 111(32.5)$ & $1.30(0.74-2.30)$ & 0.330 \\
\hline Wards (Physicians) & $19 / 130(14.6)$ & $25 / 111(22.5)$ & $0.59(0.29-1.20)$ & 0.113 \\
\hline Non-COVID-19 clinical area & $22 / 130(16.9)$ & $19 / 111(17.1)$ & $0.99(0.48-2.06)$ & 0.968 \\
\hline Non-clinical area & $18 / 130(13.9)$ & $25 / 111(22.5)$ & $0.55(0.27-1.13)$ & 0.080 \\
\hline Other area & $6 / 130(4.6)$ & $1 / 111(0.9)$ & $5.32(0.63-246.97)$ & 0.087 \\
\hline
\end{tabular}

${ }^{*} \mathrm{n}(\%)$, unless otherwise specified. OR: odds ratio; $95 \% \mathrm{Cl}$ : $95 \%$ confidence interval; IQR: interquartile range; BMI: body mass index; ICU: intensive care unit; ER: emergency room.

personnel, and physicians between cases and controls (Table 2). After adjustment for age, comorbidities, and working place, the corresponding aORs, $95 \% \mathrm{Cls}$ and $\mathrm{p}$ values were as follows: $\mathrm{aOR}=9.19$, $95 \% \mathrm{Cl}=1.05-80.22, \mathrm{p}=0.045$ (nurses in wards); $\mathrm{aOR}=4.09,95 \% \mathrm{Cl}=1.55-10.83, \mathrm{p}=0.005$ (kitchen personnel); and $\mathrm{aOR}=0.12,95 \% \mathrm{Cl}=0.03-0.54$, $\mathrm{p}=0.006$ (physicians).

The hospital epidemic curve of COVID-19 closely resembled that of the Mexico City metropolitan area in the same time period, and no discernible intrahospital clusters of COVID-19 cases were found by means of visual analysis (Fig. 1).

\section{DISCUSSION}

This study showed that certain working categories were positively (nurses, kitchen workers) or negatively (physicians) associated with being an $A C$ of SARS-CoV-2; furthermore, a tendency in nurses being diagnosed with COVID-19 was noticed (statistically non-significant finding).

In general, the data presented in this report point to the nurse as a particular risk $\mathrm{HCW}$ category in our setting, a finding that has been mentioned previously ${ }^{1}$. However, notwithstanding the obvious risk posed by close proximity to infected patients, the 
Table 2. Factors associated with being an asymptomatic carrier in bivariate analysis

\begin{tabular}{|c|c|c|c|c|}
\hline Variable & $\begin{array}{c}\text { Cases } \\
(n=37)^{*}\end{array}$ & $\begin{array}{l}\text { Controls } \\
(n=944)^{*}\end{array}$ & $\begin{array}{c}\text { OR } \\
(95 \% \mathrm{Cl})\end{array}$ & $\mathrm{p}$ \\
\hline Male sex & $20(54.1)$ & $397(42.1)$ & $1.62(0.79-3.34)$ & 0.148 \\
\hline Age, years (median, IQR) & $39(26-43)$ & $33(28-45)$ & $1.0(0.97-1.03)$ & 0.842 \\
\hline \multicolumn{5}{|l|}{ Working category: } \\
\hline Physician & $3(8.1)$ & $371(39.3)$ & $0.14(0.03-0.44)$ & $<0.001$ \\
\hline Nurse & $15(40.6)$ & $264(27.9)$ & $1.76(0.83-3.60)$ & 0.096 \\
\hline Laboratory worker & $0(0)$ & $63(6.7)$ & $0(0-1.46)$ & 0.104 \\
\hline Kitchen personnel & $8(21.6)$ & $50(5.3)$ & $4.93(1.85-11.76)$ & $<0.001$ \\
\hline Office personnel & $4(10.8)$ & $84(8.9)$ & $1.24(0.31-3.61)$ & 0.690 \\
\hline Other category & $7(18.9)$ & $112(11.9)$ & $1.73(0.63-4.15)$ & 0.197 \\
\hline \multicolumn{5}{|l|}{ Comorbidities: } \\
\hline Diabetes mellitus & $0(0)$ & $8(0.8)$ & $0(0-12.42)$ & 0.574 \\
\hline Lung disease (any) & $1(2.7)$ & $28(3.0)$ & $0.91(0.02-5.83)$ & 0.926 \\
\hline Systemic hypertension & $0(0)$ & $33(3.5)$ & $0(0-2.89)$ & 0.247 \\
\hline Cardiovascular disease & $0(0)$ & $3(0.3)$ & $0(0-33.44)$ & 0.731 \\
\hline Obesity (BMI $\geq 30$ ) & $8(21.6)$ & $150 / 922(16.3)$ & $1.42(0.55-3.26)$ & 0.390 \\
\hline Current smokers & $8(21.6)$ & $142(15.0)$ & $1.56(0.60-3.58)$ & 0.275 \\
\hline \multicolumn{5}{|l|}{ Working place: } \\
\hline ICU/ER & $10(27.0)$ & $342(36.2)$ & $0.65(0.28-1.41)$ & 0.252 \\
\hline Wards (Nurses) & $6(16.2)$ & $66(7.0)$ & $2.57(0.84-6.58)$ & 0.035 \\
\hline Wards (Physicians) & $1(2.7)$ & $100(10.6)$ & $0.23(0.0-1.43)$ & 0.121 \\
\hline Non-COVID-19 clinical area & $9(24.3)$ & $191(20.2)$ & $1.27(0.52-2.82)$ & 0.545 \\
\hline Non-clinical area & $11(29.7)$ & $193(20.4)$ & $1.65(0.72-3.52)$ & 0.172 \\
\hline Other area & $0(0)$ & $52(5.5)$ & $0(0-1.79)$ & 0.142 \\
\hline
\end{tabular}

${ }^{*} \mathrm{n}(\%)$, unless otherwise specified. OR: odds ratio; $95 \% \mathrm{Cl}$ : $95 \%$ confidence interval; IQR: interquartile range; BMI: body mass index; ICU: intensive care unit; ER: emergency room

influence of behavior and social interactions inside the hospital could not be ruled out; for instance, it was frequently observed that nurses tended to meet each other in crowded places and neglect protective regulations when outside the clinical area, although this could not be measured or compared to other groups.

The identification of kitchen workers as a factor related to ACs was due to a heightened probability of detection, since massive voluntary screening was undertaken after a COVID-19 case was diagnosed in the hospital kitchen; however, no ACs in the kitchen ultimately developed COVID-19. The rate of presymptomatic cases was much lower in this study as compared to that of another report ${ }^{9}$.

In this study, a hint as to the influence of external factors was suggested by closely resembling epidemic curves for the hospital and the community, although no firm conclusions can be further drawn. Previous studies have noted that a high proportion of COVID-19 cases in HCWs was actually related to household or community contacts $5,13-15$, and that the latter seemed to be related to higher attack rates than exposures in healthcare centers ${ }^{16}$. An observation that further supported the possible existence of external factors in this study is that COVID-19 
Figure 1. Comparison of the coronavirus disease-19 epidemic curves for Mexico City metropolitan area and the study hospital, February 22-May 21, 2020.

\section{Number of cases (Study Hospital)}

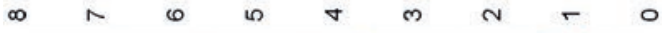
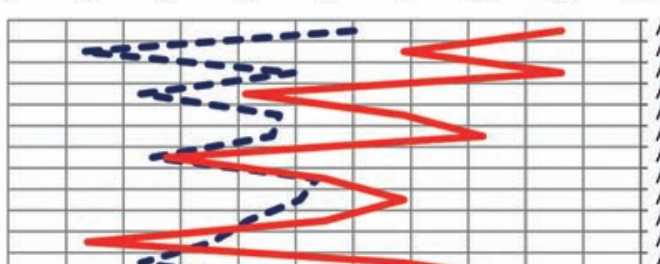

ew- $-L Z$ KeW-OZ Kew-81 New-9

KeW-ct

KeW- $\varepsilon 1$
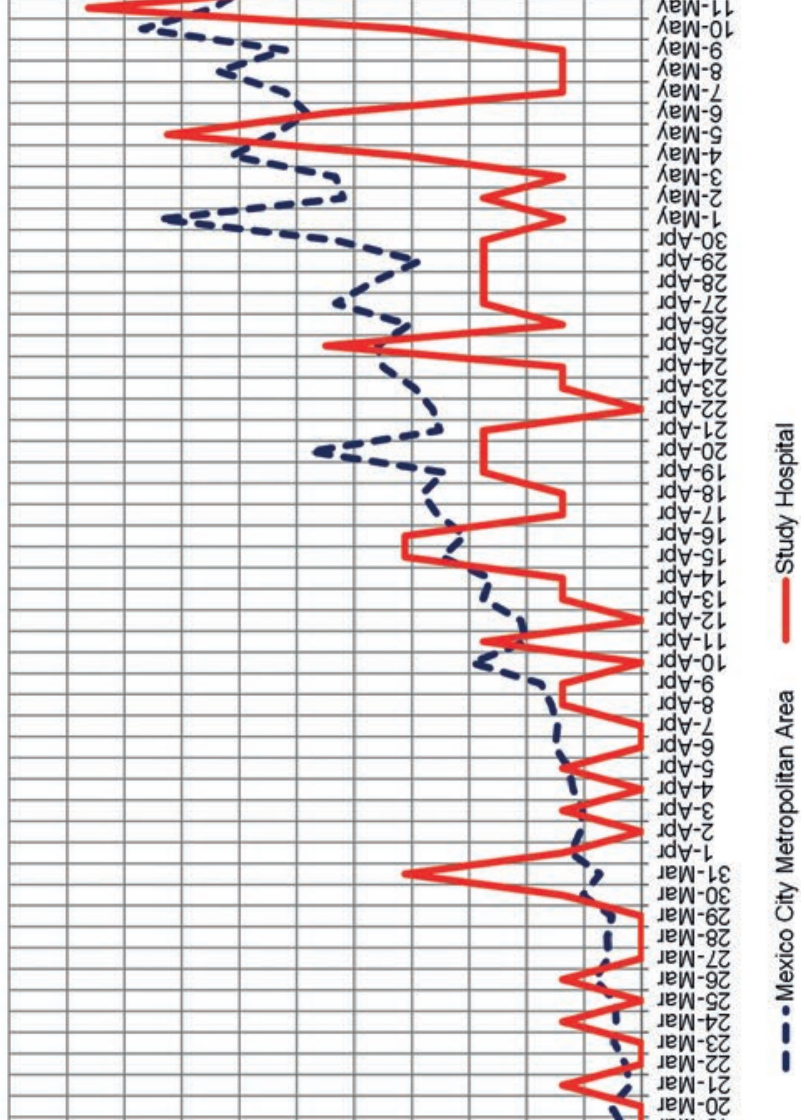

JeW-iC

JeW-0Z
JeW-6L

jew-8t

+ JeW-

נew-9l

Jew-t

- JeW-

- jew-LL

JeW-0

jeW-6
jeW-8

JeW-8

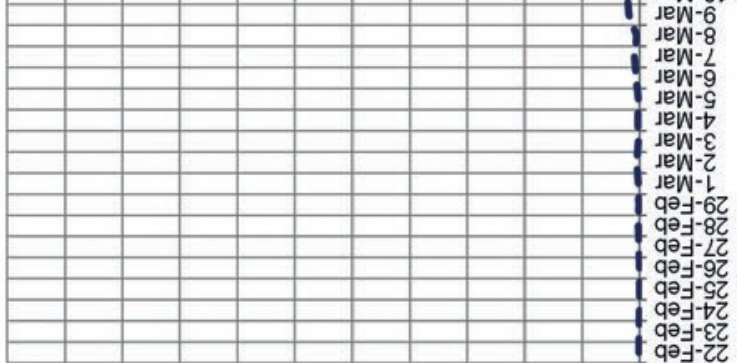

운 \& \& \&

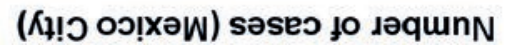


patients were admitted to our hospital a median of 7 days after symptom onset (interquartile range, 5-10 days), at a time when the majority of them would theoretically be expected to represent a low risk for intrahospital transmission ${ }^{17-19}$.

Although this study found that some of the factors associated with being a case or an AC are consistent with other reports, social and behavioral factors unaccounted for are undoubtedly intermingled, and further studies will aid to better define their importance.

We acknowledge limitations. First, the study sample was not random and included consecutive subjects in a defined time frame. Second, generalization is not possible due to important differences in the pandemic behavior across countries, regions, and hospitals. Third, although the adherence rate to hand hygiene and proper use of personal protective equipment was not measured, distribution of kits to HCWs as mentioned above was universal, purportedly facilitating $\mathrm{HCW}$ prevention behaviors when in contact with patients, although behavior beyond the clinical area was not measured. Two major strengths of this study are the complete assessment and follow-up of the included subjects, providing a reliable categorization of health status, and the exhaustive testing of all subjects, which provides a precise estimate of the initial behavior of the COVID-19 epidemic in this Mexican hospital.

In summary, $\mathrm{HCW}$ category was the predominant factor associated with being an AC of SARS-CoV- 2 in this study, and being a COVID-19 case tended to be more frequent in nurses, although this finding was not statistically significant in multivariable analysis.

\section{REFERENCES}

1. Lai X, Wang M, Qin C, Tan L, Ran L, Chen D, et al. Coronavirus disease 2019 (COVID-2019) infection among health care workers and implications for prevention measures in a tertiary hospital in Wuhan, China. JAMA Netw Open. 2020;3:e209666.

2. Nguyen LH, Drew DA, Graham MS, Joshi AD, Guo CG, Ma W, et al. Risk of COVID-19 among front-line health-care workers and the general community: a prospective cohort study. Lancet Public Health. 2020;5:e475-83.

3. Information Related to COVID-19 Cases in Mexican Healthcare Workers. Mexico: Health Secretariat; 2020. Available from: https://www.gob.mx/cms/uploads/attachment/file/595257/ covid-19_personal_de_salud_2020.11.23.pdf. [Last accessed on 2020 Nov 23].

4. Daily Technical COVID-19 Communication. Mexico: Health Secretariat; 2020. Available from: https://www.gob.mx/cms/uploads/attachment/file/594103/comunicado_tecnico_diario_ covid-19 2020.11.23.pdf. [Last accessed on 2020 Nov 23].

5. Rivett L, Sridhar S, Sparkes D, Routledge M, Jones NK, Forrest $\mathrm{S}$, et al. Screening of healthcare workers for SARS-CoV-2 highlights the role of asymptomatic carriage in COVID-19 transmission. Elife. 2020;9:e58728.

6. Vahidy FS, Bernard DW, Boom ML, Drews AL, Christensen P, Finkelstein J, et al. Prevalence of SARS-CoV-2 infection among asymptomatic health care workers in the greater Houston, Texas, Area. JAMA Netw Open. 2020;3:e2016451.

7. Folgueira MD, Muñoz-Ruiperez C, Alonso-López MA, Delgado R. SARS-CoV-2 Infection in Health Care Workers in a Large Public Hospital in Madrid, Spain, during March 2020, medRxiv; 2020. Available from: https://www.medrxiv.org/content. [Last accessed on 2020 Sep 15].

8. Ran L, Chen X, Wang Y, Wu W, Zhang L, Tan X. Risk factors of healthcare workers with Coronavirus disease 2019: a retrospective cohort study in a designated hospital of Wuhan in China. Clin Infect Dis. 2020;71:2218-21.

9. Yang R, Gui X, Xiong Y. Comparison of clinical characteristics of patients with asymptomatic vs symptomatic Coronavirus disease 2019 in Wuhan, China. JAMA Netw Open. 2020;3: e2010182.

10. Steensels D, Oris E, Coninx L, Nuyens D, Delforge ML, Vermeersch $P$, et al. Hospital-wide SARS-CoV-2 antibody screening in 3056 staff in a tertiary Center in Belgium. JAMA. 2020; 324:195-7.

11. Corman VM, Landt O, Kaiser M, Molenkamp R, Meijer A, Chu DK, et al. Detection of 2019 novel Coronavirus (2019-nCoV) by real-time RT-PCR. Euro Surveill. 2020;25:2000045.

12. COVID-19 Epidemiological Data for Mexico. Mexico: General Direction of Epidemiology, Health Secretariat; 2020. Available from: https://www.covid19.sinave.gob.mx. [Last accessed on 2020 Jul 29].

13. Hunter E, Price DA, Murphy E, van der Loeff IS, Baker KF, Lendrem D, et al. First experience of COVID-19 screening of healthcare workers in England. Lancet. 2020;395:e77-8.

14. Kluytmans-van den Bergh MF, Buiting AG, Pas SD, Bentvelsen RG, van den Bijllaardt W, van Oudheusden AJ, et al. Prevalence and clinical presentation of health care workers with symptoms of Coronavirus disease 2019 in 2 Dutch hospitals during an early phase of the pandemic. JAMA Netw Open. 2020;3:e209673.

15. World Health Organization. Report of the WHO-China Joint Mission on Coronavirus Disease 2019 (COVID-19). Geneva: World Health Organization; 2020. Available from: https://www. who.int/publications/i/item/report-of-the-who-china-jointmission-on-coronavirus-disease-2019-(covid-19). [Last accessed on 2020 Jul 01].

16. Klompas M, Baker MA, Rhee C. Airborne transmission of SARSCoV-2: theoretical considerations and available evidence. JAMA. 2020;;324:441-2.

17. Garg S, Kim L, Whitaker M, O'Halloran A, Cummings C, Holstein $\mathrm{R}$, et al. Hospitalization rates and characteristics of patients hospitalized with laboratory-confirmed Coronavirus disease 2019-COVID-NET, 14 States, March 1-30, 2020. MMWR Morb Mortal Wkly Rep. 2020;69:458-64.

18. Wölfel R, Corman VM, Guggemos W, Seilmaier M, Zange S, Müller MA, et al. Virological assessment of hospitalized patients with COVID-19. Nature. 2020;581:465-9.

19. Cheng HY, Jian SW, Liu DP, Ng TC, Huang WT, Lin HH. Contact tracing assessment of COVID-19 transmission dynamics in Taiwan and risk at different exposure periods before and after symptom onset. JAMA Intern Med. 2020;180:1156-63. 